\title{
Fuzzy graph theory applied to brittle plane network- A need for carbon sequestration models
}

\author{
O.T. Manjusha ${ }^{1}$, Soumyajit Mukherjee ${ }^{2 *}$ \\ 1. Department of Mathematics \\ Govt. Arts and Science College, Kondotty \\ Malappuram 673 041, Kerala, INDIA \\ 2. Department of Earth Sciences \\ Indian Institute of Technology Bombay \\ Powai, Mumbai 400 076, Maharashtra, INDIA
}

*Author for correspondence: soumyajitm@gmail.com, smukherjee@iitb.ac.in

Statement: This article is a non-peer reviewed preprint, submitted to EarthArXi. The article has been submitted to the Journal of Geosciences Research (Formerly Gondwana Geological Magazine Journal) for peer review. 


\title{
Fuzzy graph theory applied to brittle plane network- A need for carbon sequestration models
}

\author{
O.T. Manjusha ${ }^{1}$, Soumyajit Mukherjee ${ }^{2 *}$ \\ 1. Department of Mathematics \\ Govt. Arts and Science College, Kondotty \\ Malappuram 673 041, Kerala, INDIA \\ 2. Department of Earth Sciences \\ Indian Institute of Technology Bombay \\ Powai, Mumbai 400 076, Maharashtra, INDIA \\ *Author for correspondence: soumyajitm@gmail.com,smukherjee@iitb.ac.in
}

\begin{abstract}
Improved carbon sequestration (CCS) models with rocks as sinks require incorporation of uncertainty into the models. In such cases of uncertain geoscientific problems, fuzzy graph theory can be useful. Brittle shear plane network with indistinct shear planes is common in natural sheared rocks, and can be targeted for CCS. Due to non-unique possibility of continuity of P-planes, it is not possible to represent such networks as crisp graphs. We present few natural examples of the former type of P-planes in shear zones, and how fuzzy graph theory can represent the fracture network and fluid flow. The process involves assigning some sample numerical probability to represent the connectedness between the underdeveloped Pplanes and the Y-planes. The presentation is a geometric exercise and does not extend to the genesis of the shear zones.
\end{abstract}

(Words: 131)

Keywords: Network; fluid flow; structural geology; shear planes; geoscientific uncertainty; waste disposal 


\section{Introduction}

Structural geological modeling requires representation of structures into some numerical form. When the structures are clearly decipherable, this is rather easy, for example, one can apply the graph theory (e.g., Sanderson et al., 2018; Mukherjee 2019). Classical graph theory works when there is a full certainty that which nodes are connected by which vertices through edges.

Various brittle plane geometries develop in rocks at shallow crustal depths that undergo brittle shear (Fig. 1a). In case the shear planes are clearly delineated (Fig. 1b), one can use graph theory to represent the brittle plane of sigmoid shear bound by parallel planar shear planes are commonly seen in sheared rocks.

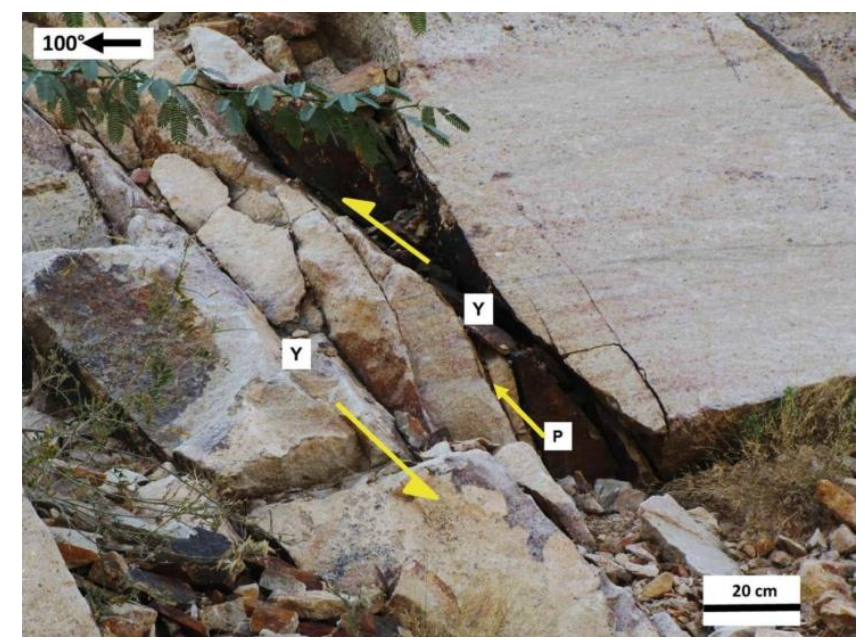

Fig. 1a. Clearly visible $Y$ and $P$ planes. Top-to-ESE brittle shear with distinct $Y$ - and $P$ planes within fluvial sandstone of the Sarnoo hill area, observed on a sub-vertical section. Location: $W$ of Sarnoo hill area, SE of the Sarnoo village, Barmer basin, India. Reproduced from fig. 1.18 of Mukherjeeet al. (2020). 


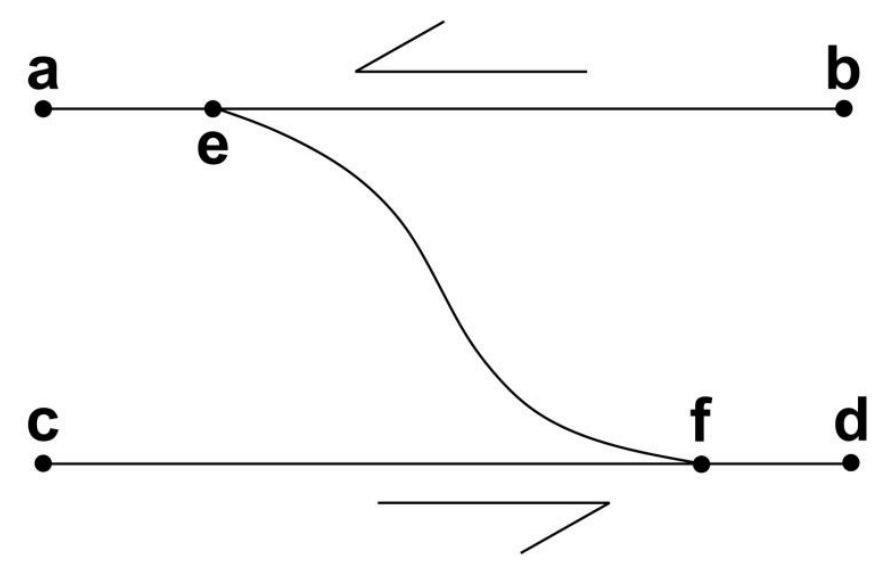

Fig. 1b. A brittle plane network in a brittle shear zone. Reproduced from fig. $2 c$ of Mukherjee (2019). Given that its nodes are $a, b, c, d$, e and $f$, the adjacency matrix is:

$\begin{array}{lllllll} & a & b & c & d & e & f \\ a & 0 & 0 & 0 & 0 & 1 & 0 \\ b & 0 & 0 & 0 & 0 & 0 & 1 \\ c & 0 & 0 & 0 & 0 & 0 & 1 \\ d & 0 & 0 & 0 & 0 & 0 & 1 \\ e & 0 & 0 & 0 & 0 & 0 & 1 \\ f & 0 & 0 & 1 & 1 & 0 & 0\end{array}$

Carbon sequestration (CCS) has recently been modeled in terms of extraneous $\mathrm{CO}_{2}$ sources and $\mathrm{CO}_{2}$ sinks in rocks and P-graph modeling approach has been undertaken (Chong et al. 2014). Fractured rocks especially coal has been targeted for CCS (e.g., Chen et al. 2020). But since connectivity of fractures is uncertain at depth, establishing models on CCS becomes problematic (fig. 1 of Zhang et al. 2009). Pashin et al. (2008) presented a discrete fracture network model for CCS in coal. Such models would be possible when existence of the sink locations in the coal is fairly well understood. When the existence of the sinks is uncertain, models will nevertheless be required to be built in decision making. Other uncertainties can be "unproven nature" of the carbon sequestration technology, economic cost and life cycles of the CCS technologies and so on (Tan et al., 2010 and references therein). Researchers have 
identified CCS as one of the potential areas where graph theory concept needs more elaboration (e.g., Farid et al., 2021).

Anand et al. (2021) discussed various kinds of uncertainties in parameters in structural geological problems. The sigmoid planes can be indistinct due to (i) poor snap quality, (ii) they are indeed developed less prominently, or (iii) sometimes a part of them is below the ground surface hence not seen. For example, in seismic images, brittle planes may not always be clearly decipherable. This can be because of such brittle planes are below the seismic resolution (Misra and Mukherjee 2018), and yet the modeler needs to make some presentation of the brittle plane network. In such cases, where the classical graph theory cannot represent the brittle plane network, fuzzy graph theory should be used.

The concept of fuzzy sets was introduced by Zadeh (1965), which later was well applied to solve several research problems that are uncertain in nature. Crisp set is a well-defined collection of distinct objects. If there is any vagueness in the description of objects, the concept of fuzzy set is used. In other words, a fuzzy set is used to represent qualitative data. The crisp set is not able to work with scientific problems with inherent uncertainty because it consists of just two truth values: 0 - false and 1-true. These single values of membership degree are unable to manage the uncertainties. Atanassov (1999) proposed the intuitionistic fuzzy set to manage uncertain situations using an extra degree of membership, defined as the hesitation margin. Intuitionistic fuzzy set is as an extension of Zadeh's theory of fuzzy sets. Compared to classical fuzzy set it is more flexible and efficient to work with uncertainty due to the presence of hesitation margin. 


\section{Example}

Figs. 2a-c present a network of brittle shear planes where not all P-planes can be tracked with confidence. Here the curved P-plane AB joins two sub-parallel Y-planes. However, the other P-planes (CD, KZ, EF, GH and IJ) and do not join both the C-

planes. In some cases the P-planes are too close-spaced in order to distinguish them confidently in naked eyes. A simplified situation can be thought in this case (Fig. 3). 

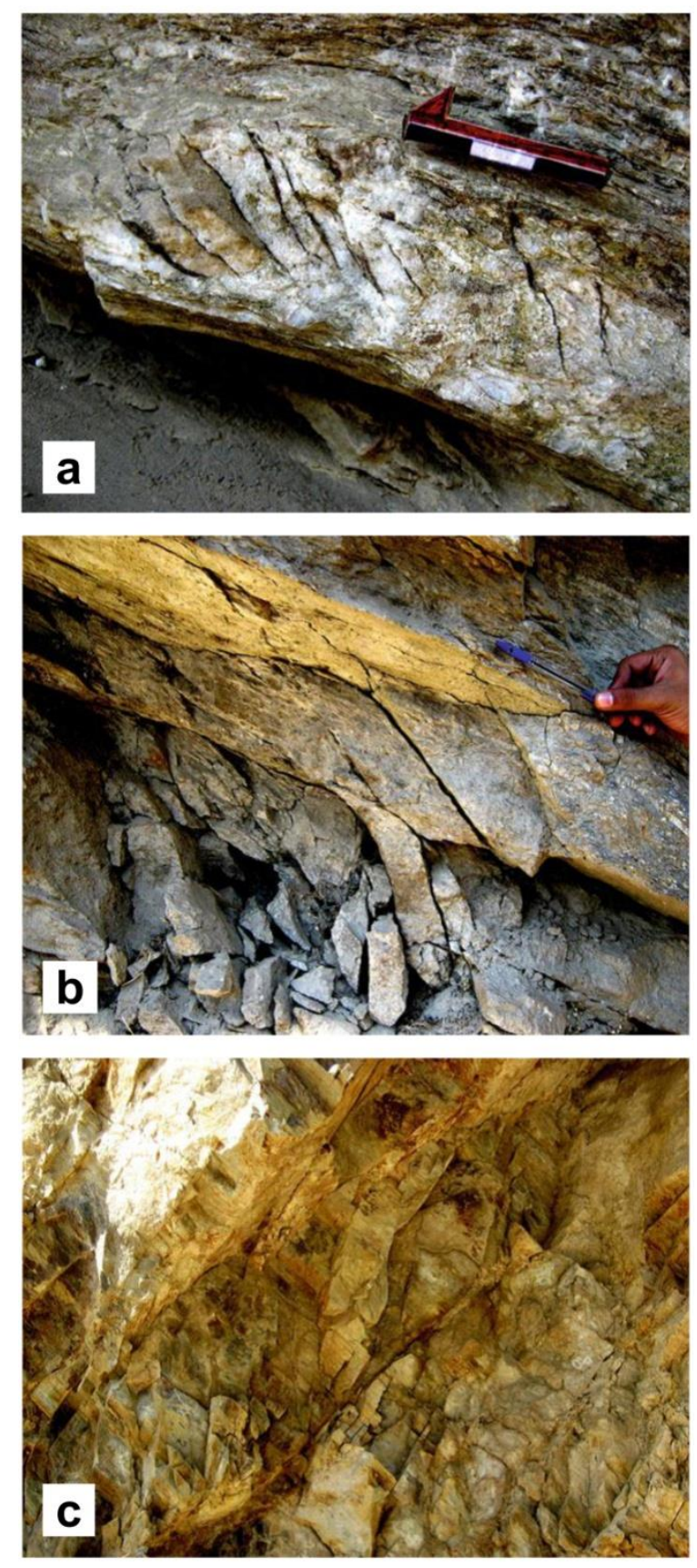

Fig.2. Brittle plane network consisting of $Y$ and $P$ planes. Here some of these planes cannot be traced continuously with full confidence, and hence acrisp graph cannot be visualized. Fuzzy graph theory can be applied in these cases. All examples from mylonitized gneiss in Greater Himalayan Crystallines, Bhagirathi section, Uttarakhand, India- (a,b) Top-to-left (up) shear. India; reproduced from figs. 5.15 and 5.20, respectively, of Mukherjee (2014). (c) Top-to-right (up) shear; reproduced from fig. 5.21 of Mukherjee (2014). 


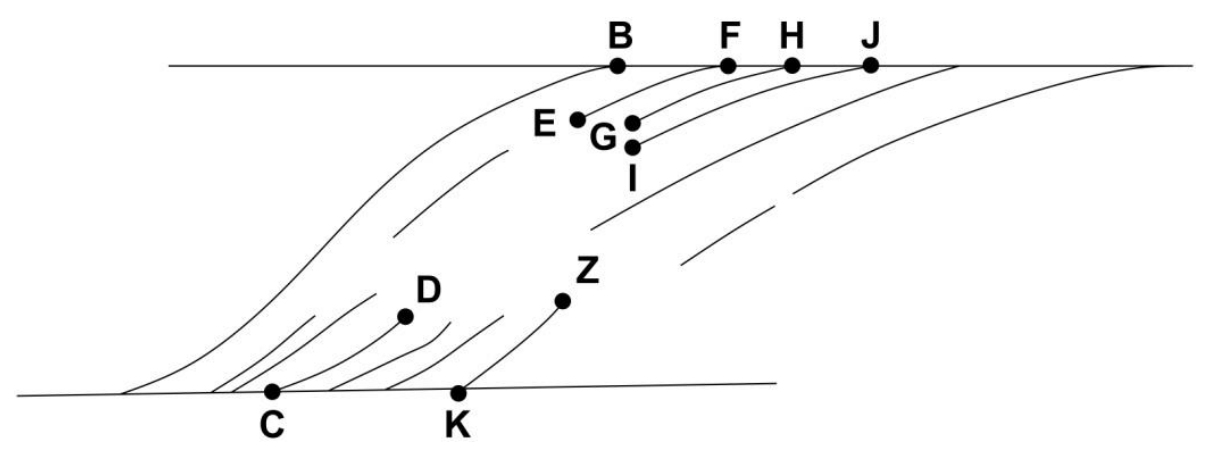

Fig. 3. An idealized brittle shear plane network. Few sigmoid P-planes (e.g., AB) join the C-planes. The other P-plane, CD, KZ, EF, GH and IJ, are not fully developed and merge with just a single C-plane. In such a situation, fuzzy graph theory has been applied in this work.

We represent fracture network using intuitionistic fuzzy graph as follows (Fig. 4). Points (nodes) $\mathrm{A}$ and $\mathrm{B}$ are certainly connected with $\mathrm{AB}$ as an arc. But it is uncertain for some reason whether point D connects with E, G or I by some arc. Connection between D and E or I therefore can be represented as an intutionistic fuzzy graph. Now if by zooming the image or by some image enhancement technique, a connection between $\mathrm{D}$ and $\mathrm{G}$ is established with some degree of confidence, it means that there is a much higher probability/possibility that they are connected, and therefore connections between D and E or D and I would be less likely/less probable. Putting some sample numerical probability values in a scale of 0 ("certainly not") to 1 ("certainly"), or using intuitionistic fuzzy graph enables one to represent the fracture network. In this graph, take the node set as $\mathrm{V}=\{\mathrm{A}, \mathrm{B}, \mathrm{C}, \mathrm{D}, \mathrm{E}, \mathrm{F}, \mathrm{G}, \mathrm{H}, \mathrm{I}, \mathrm{J}, \mathrm{K}, \mathrm{Z}\}$ and $\mathrm{A}(0.9,0.1)$ denotes the degree of membership and degree of non-membership of the node $A$ and similarly for all other nodes The ordered pair $(0.7,0.1)$ denotes the degree of membership and degree of nonmembership of the arc relation $e_{F H}=(F, H)$ on $V \times V$ and so on. These numerical values of possibilities are given according to the degree of confidence of the connection. 
We will now get into further detail of fuzzy graph theory to tackle flow problems. The adjacency matrix of a fuzzy graph $G:(V, \sigma, \mu)$ is an $n \times n$ matrix defined as $A=\left[a_{i j}\right]$ where $a_{i j}=\mu\left(v_{i}, v_{j}\right)($ Anjali and Mathew 2013).

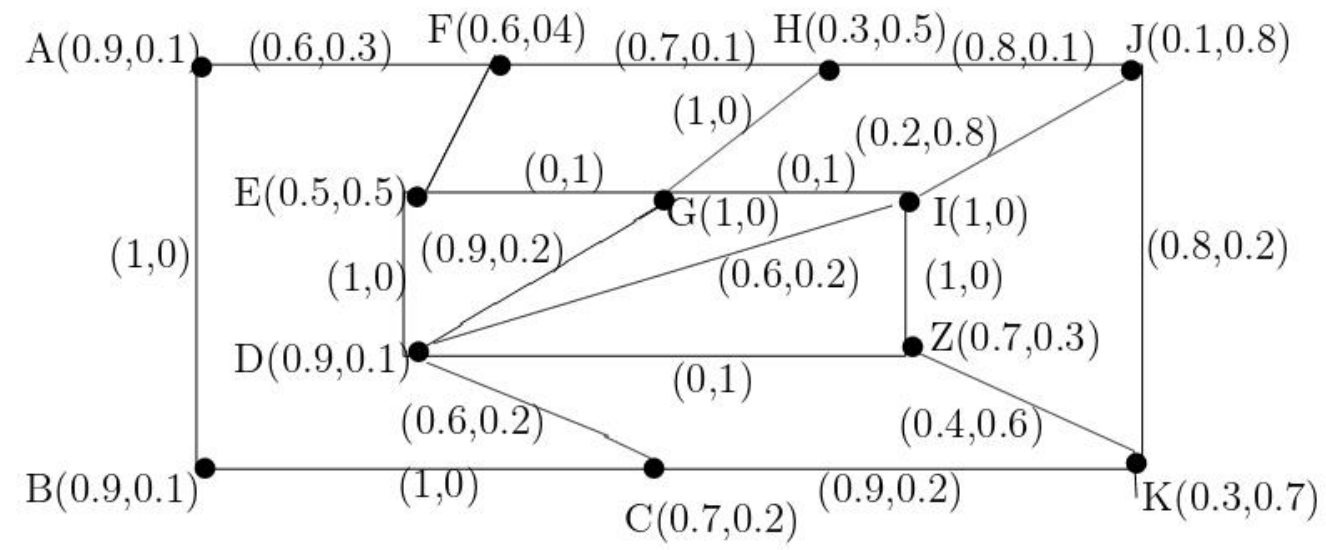

Fig. 4. A fuzzy graph corresponding to the case of Fig. 3.

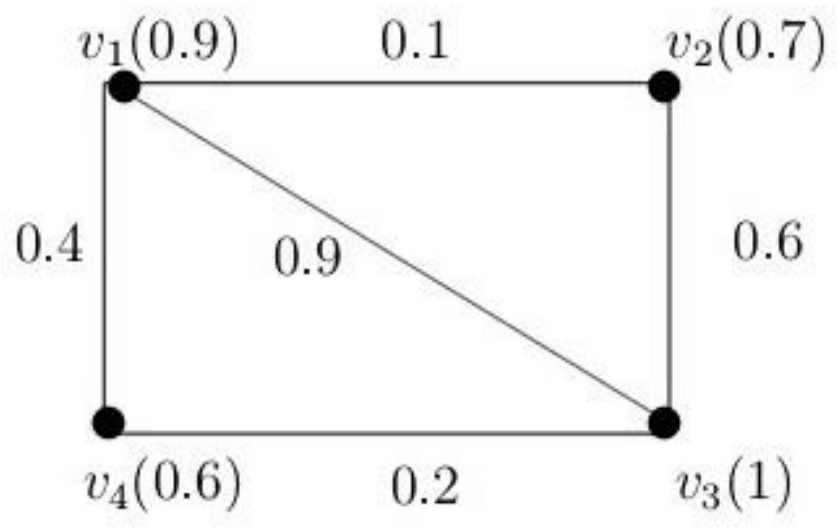

Fig. 5. A fuzzy graph with nodes $v_{1}, v_{2}, v_{3}$ and $v_{4}$. Values inside brackets of the nodes are the fuzzy weights of the respective nodes. Fuzzy arc weights are represented by the values 0.1, 0.2, 0.4, 0.6, and 0.9. 
For example, consider the fuzzy graph in Fig 5. Its adjacency matrix is:

$v_{2}\left[\begin{array}{cccc}v_{1} & v_{2} & v_{3} & v_{4} \\ v_{3} & 0.1 & 0.9 & 0.4 \\ 0.1 & 0 & 0.6 & 0 \\ v_{4} & 0.9 & 0 & 0.2 \\ 0.4 & 0 & 0.2 & 0\end{array}\right]$

In this fuzzy graph, the numbers inside the first bracket represents the node weights, which do not appear in the adjacency matrix since. Instead only the arc weights are used for the entries in the adjacency matrix. Such a presentation is as per Anjali and Mathew (2013). The entries in the adjacency matrix are obtained as follows. Since $v_{1}$ is not shown to be connected with itself by any edge, the entry is 0 . Since $v_{1}$ is adjacent with $v_{2}$ with arc weight 0.1 , the entry is 0.1 and so on.

Consider the network in Fig. 6. From $v_{2}$ to $v_{1}$ the flow can vary in magnitude from 7 to 2 units. Therefore we take fuzzy weights 0.7 for $v_{2}$ and 0.2 for $v_{1}$. Similarly, one can take the fuzzy weight of $v_{3}$ as $0.3, v_{4}$ as 0.1 . This is a first-time approach through this work. Suppose the fuzzy graph presented in Fig 6 has no arrows on edges. Then its corresponding adjacency matrix will differ. 


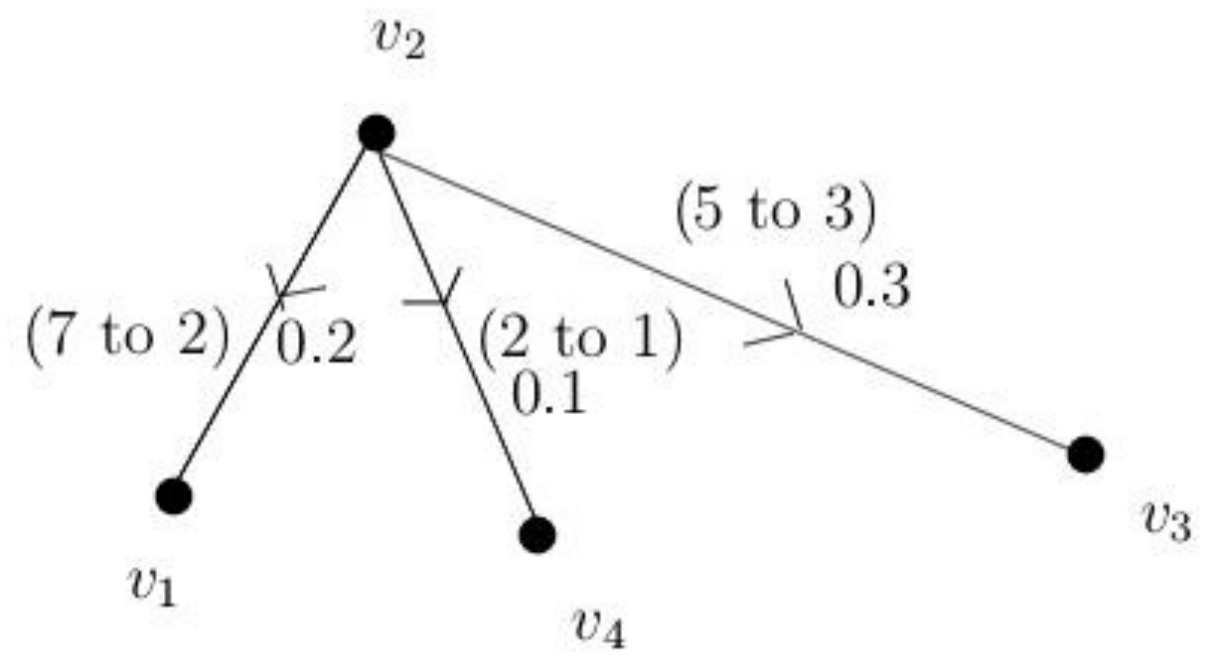

Fig. 6. A network, from nodes $v_{2}$ to $v_{1}$ the flow can vary in magnitude from 7 to 2 units. Therefore, we take fuzzy weights 0.7 for $v_{2}$ and 0.2 for $v_{1}$. Similarly, one can take the fuzzy weight of $v_{3}$ as $0.3, v_{4}$ as 0.1 .

That means flow can happen from one vertex to another and also vice versa. The definition of a fuzzy graph allows us to give the arc weights in the following way. The arc (edge) weights are taken as the minimum among the corresponding node weights. This is logically true, since the maximum flow between two nodes is the minimum capacity among the two nodes. In that case, the adjacency matrix is:

\begin{tabular}{|c|c|c|c|c|c|}
\hline & & $v_{1}$ & $v_{2}$ & $v_{3}$ & $v_{4}$ \\
\hline & $v_{1}[$ & 0 & 0 & 0 & 0 \\
\hline $\mathrm{A}=$ & $v_{2}$ & 0.2 & 0 & 0.3 & 0.1 \\
\hline & $v_{3}$ & 0 & 0 & 0 & 0 \\
\hline & $v_{4}$ & 0 & 0 & 0 & 0 \\
\hline
\end{tabular}

Suppose, instead of a range of flow, definite units of flow were given (e.g., 7, 5 and 2). We then assign fuzzy weights as $0.7,0.5$ and 0.2 , respectively, for arcs. If a range of flow is given, 
we will get a freedom to take the minimum value amongst the range for representing the arc weight for the range of flow. As per the definition of adjacency matrix, one can represent these values by using their corresponding fuzzy weights. If 2,1 and 3 are avoided, i.e., flow range 7 to 2 becomes 7, flow range 2 to 1 becomes 2 and flow range 5 to 3 becomes 5 , the flow becomes exact, and one can assign the fuzzy weights. In fuzzy graph, we can give the maximum value of node weight and arc weight as 1 and minimum as 0 . All other values are in between 1 and 0 . A value equal to 1 means that the flow has fully happened. The intensity of the flow is represented by using these fuzzy weights.

Ramakrishnan and Lakshmi (2008) discussed how to fuse two nodes of the same fuzzy graph as follows. Let $G$ : $(V, \sigma, \mu)$ be a fuzzy graph and let $u, v \in \sigma$. By the join (fusion) of two vertices $u$ and $v$, the following is meant.

(i) Fuse (join) the vertices $u$ and $v$ as $u v$ in the corresponding crisp graph $G^{*}=\left(\sigma^{*}, \mu^{*}\right)$ and then consider its underlying simple graph. A simple graph is the one without loops and possessing multiple edges.

(ii) The resulting fuzzy graph is $G_{u v}=\left(\sigma_{u v}, \mu_{u v}\right)$ where

$$
\begin{aligned}
& \sigma_{u v}(x)=\{\max [\sigma(u), \sigma(v)] \text { if } x=u v \\
& \sigma(x) \text { if } x \neq u v\} \\
& \text { and } \mu_{u v}(x, y)=\{\max [\mu(x, u), \mu(x, v)] \text { if } y=u v \\
& \max [\mu(u, y), \mu(v, y)] \text { if } x=u v \\
& \mu(x, y) \text { if } x \neq u v, y \neq u v\}
\end{aligned}
$$

Consider the fuzzy graph in Fig. 7. Fig. 8 represents the fusing of the vertices $a$ and $b$. 


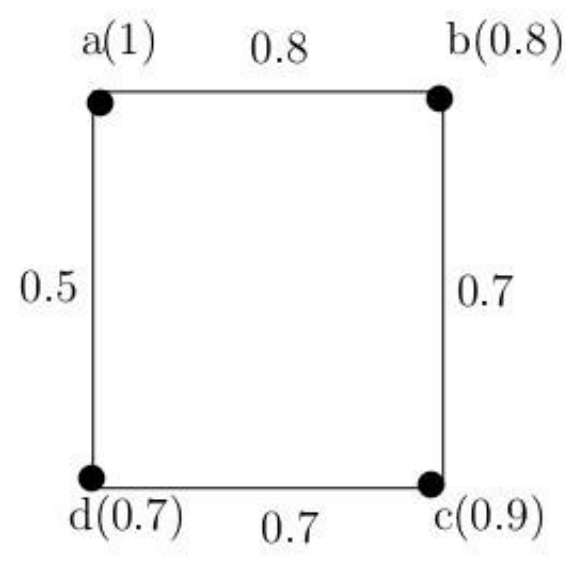

Fig. 7. Example of a fuzzy graph with nodes $a, b, c$ and $d$. Values inside the brackets of the nodes represent the fuzzy weights. Fuzzy arc weights are 0.5, 0.7 and 0.8.

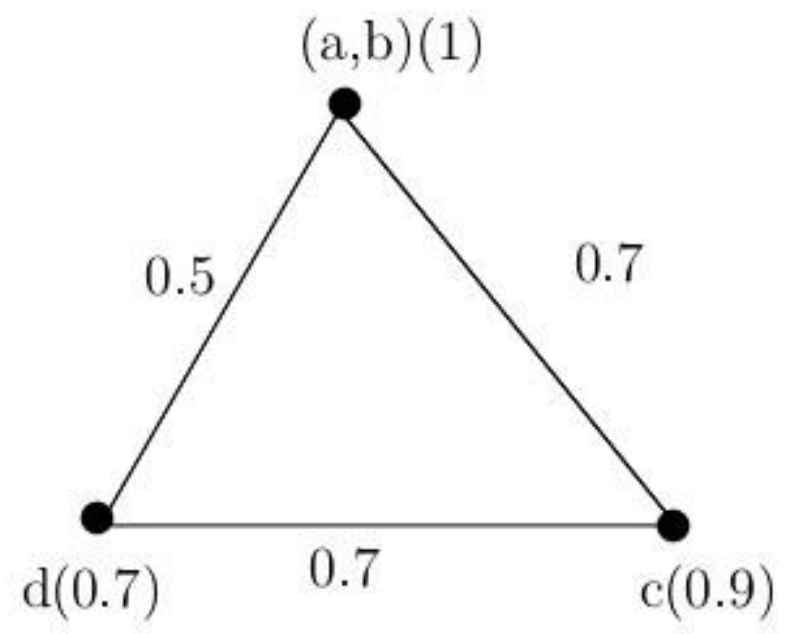

Fig. 8. Fusing nodes $a$ and $b$ in the graph presented in Fig. 7.

For instance,

$$
\begin{gathered}
\mu_{a b}(a b, d)=\operatorname{Max}\{\mu(a, d), \mu(b, d)\}=\operatorname{Max}\{0.5,0\}=0.5 \\
\sigma_{a b}(a b)=\operatorname{Max}\{\sigma(a), \sigma(b)\}=\operatorname{Max}\{1,0.8\}=1
\end{gathered}
$$


By using this idea, we can define the fusion of two vertices from two fuzzy graphs (Fig. 9).

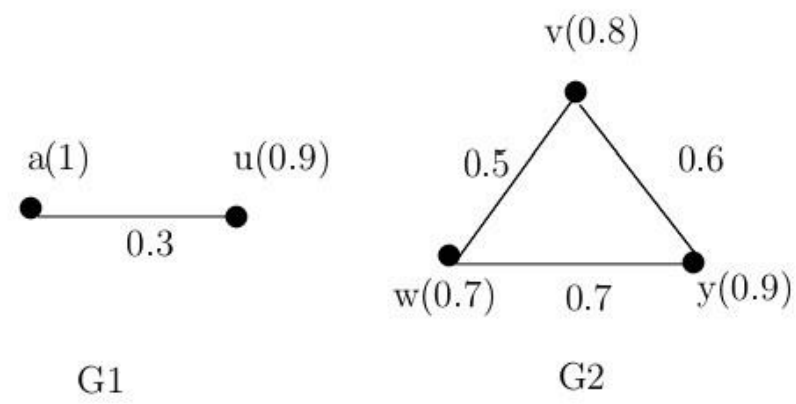

Fusing the nodes $\mathrm{u}$ and $\mathrm{v}$ we get

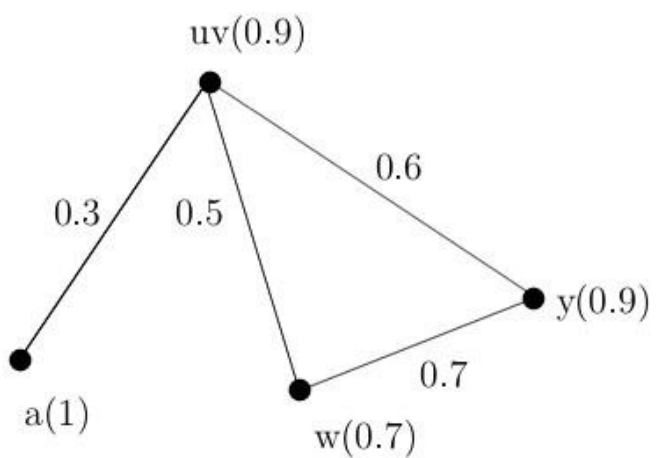

Fig. 9. Fusion of two vertices $u$ and $v$ from two fuzzy graphs $G_{1}$ and $G_{2}$, respectively into $u v$. The fused vertex is assigned a weight 0.9, which is the maximum weight in between $u$ and $v$.

Consider two fuzzy graphs $\mathrm{G}_{1}:\left(V_{1}, \sigma_{1}, \mu_{1}\right)$ and $\mathrm{G}_{2}:\left(V_{2}, \sigma_{2}, \mu_{2}\right)$. Let $u \in G_{1}, v \in G_{2}$. Fuse (join) the vertices $u$ and $v$ as $u v$ in the corresponding crisp graph and then consider the underlying crisp simple graph. The resulting fuzzy graph is $G_{u v}=\left(\sigma_{u v}, \mu_{u v}\right)$ where:

$$
\begin{gathered}
\sigma_{u v}(x)=\left\{\max \left[\sigma_{1}(u), \sigma_{2}(v)\right] \text { if } x=u v\right. \\
\sigma_{1}(x) \text { if } \quad x \in G_{1}, x \neq u v \\
\left.\sigma_{2}(x) \text { if } x \in G_{2,}, x \neq u v\right\}
\end{gathered}
$$




$$
\begin{aligned}
& \mu_{u v}(x, y)=\left\{\mu_{1}(x, u) \text { if } y=u v, x \in G_{1}\right. \\
& \mu_{2}(x, v) \text { if } y=u v, x \in G_{2} \\
& \mu_{1}(x, y) \text { if } x, y \in G_{1} \\
& \left.\mu_{2}(x, y) \text { if } x, y \in G_{2}\right\}
\end{aligned}
$$

Coming to the case of a directed fuzzy graph or a fuzzy digraph, consider the network in Fig 10.
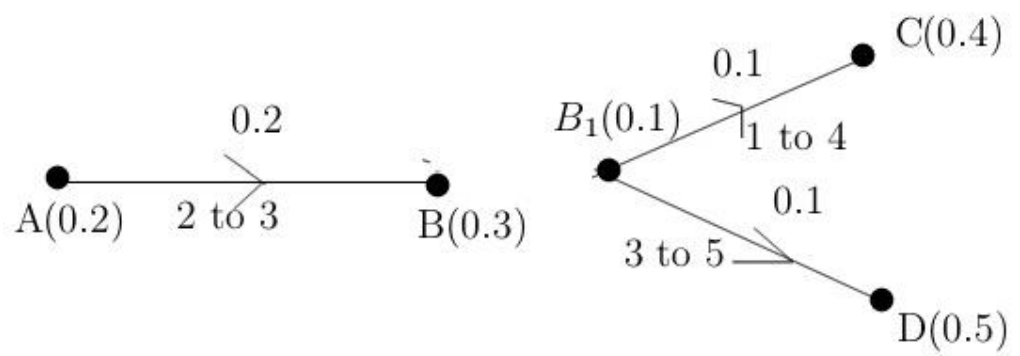

If $B$ and $B_{1}$ fused as a single vertex we get

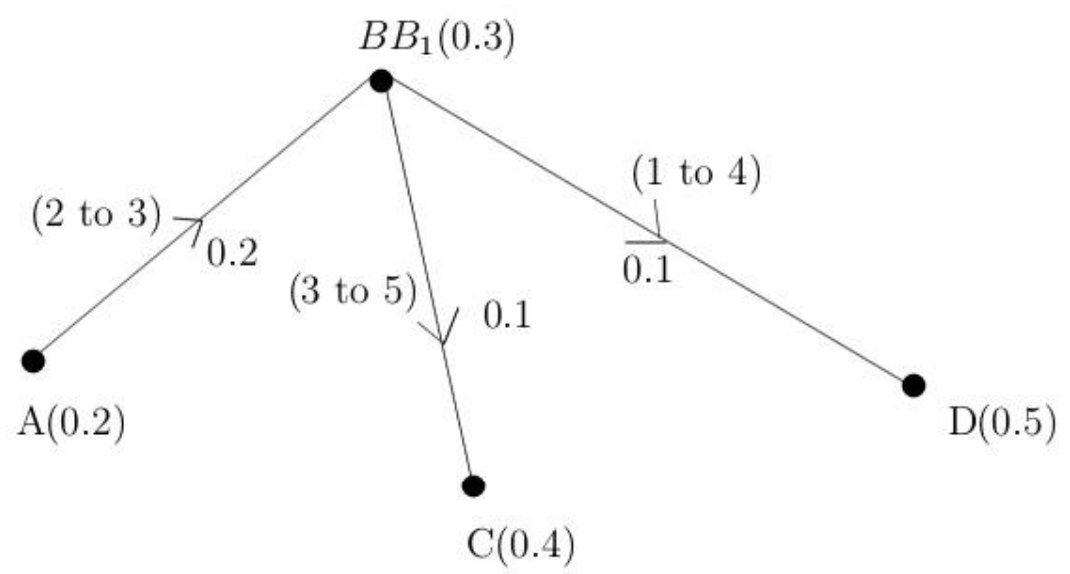

Fig. 10. Fusing two nodes $B$ and $B_{1}$ from two different networks. In the BB1 fused vertex, 0.3 is stated inside bracket. The magnitude is the maximum out of $B(0.3)$ and B1 (0.1). 
The adjacency matrix of this new fuzzy graph is given below.

$B B_{1}\left[\begin{array}{cccc}\mathrm{A} & B B_{1} & \mathrm{C} & \mathrm{D} \\ 0 & 0.2 & 0 & 0 \\ \mathrm{C}\left[\begin{array}{cccc}0 \\ 0\end{array}\right. & 0 & 0.1 & 0.1 \\ 0 & 0 & 0 & 0\end{array}\right]$

In the BB1 fused vertex, 0.3 is stated inside bracket. This means that the maximum out of B (0.3) and B1 (0.1) to be stated: "BB1 (0.3)". For the maximum taken, the logic is that the capacity elevates when nodes are fused (Ramakrishnan and Lakshmi 2008).

The general expression for fusing $n$ number of vertices is defined as follows. Fusing of $n$ number of nodes within the same fuzzy graph: Let $G=(V, \sigma, \mu)$ be a fuzzy graph and let $u_{1}, u_{2}, \ldots . . u_{n} \in \sigma^{*}$. By the join of $n$ vertices $u_{1}, u_{2} \ldots, u_{n}$ we mean:

(i) Fuse the vertices $u_{1}, u_{2} \ldots \ldots, u_{n}$ in the corresponding crisp graph $G^{*}=\left(\sigma^{*}, \mu^{*}\right)$

(ii) The resulting fuzzy graph is

$G_{u_{1} u_{2} \ldots . . u_{n}}=\left(\sigma_{u_{1} u_{2}} \ldots \ldots \ldots u_{n}, \mu_{u_{1} u_{2} \ldots \ldots . . . u_{n}}\right)$

Here

$$
\begin{gathered}
\sigma_{u_{1} u_{2} \ldots \ldots u_{n}}(x)=\left\{\max \left[\sigma\left(u_{1}\right), \sigma\left(u_{2}\right), \ldots \ldots \sigma\left(u_{n}\right)\right]\right. \\
\text { if } x=u_{1} u_{2} \ldots \ldots . u_{n} \\
\left.\sigma(x) \text { if } x \neq u_{1} u_{2} \ldots \ldots . u_{n}\right\}
\end{gathered}
$$


and

$$
\mu_{u_{1} u_{2} \ldots \ldots \ldots u_{n}}(x, y)=\left\{\operatorname { m a x } \left[\mu\left(x, u_{1}\right), \mu\left(x, u_{2}\right), \ldots \ldots \mu\left(x, u_{n}\right]\right.\right.
$$

if $y=u_{1} u_{2} \ldots \ldots . u_{n}$

$$
\begin{gathered}
\max \left[\mu\left(u_{1}, y\right), \mu\left(u_{2}, y\right) \ldots \ldots \mu\left(u_{n}, y\right)\right] \\
\text { if } x=u_{1} u_{2} \ldots \ldots u_{n} \\
\mu(x, y) \\
\text { if } \left.x \neq u_{1} u_{2} \ldots \ldots u_{n}, y \neq u_{1} u_{2} \ldots \ldots u_{n}\right\}
\end{gathered}
$$

\section{Discussions and Conclusions}

Indistinct or poorly developed brittle plane networks are common in rocks. Their numerical representation can be important for modelers, such as in fluid flow, CCS and hydrocarbon reservoir studies. As the hydrocarbon resources are depleting, flow models realistic to geologic cases are of paramount importance. In this article we introduce the concept of fuzzy graph theory as a first step to fulfill such a far-reaching aim. An example of brittle shear planes consisting of Y and P-planes was considered with different degrees of connection between them. We further presented theoretical issues regarding fuzzy digraphs and joining/fusion of vertices.

\section{Acknowledgements}

\section{CPDA grant supported SM.}

\section{References}

Anand P, Chakraverty S, Mukherjee S. 2021. Fuzzy set concept in structural geology: Example of ductile simple shear. Journal of Earth System Science 130, 193. 
Anjali N, Mathew S 2013 Energy of a fuzzy graph. Annals of Fuzzy Mathematics and Informatics, ISSN: 2093-9310.

Atanssov K T 1999 Intuitionistic Fuzzy Sets Springer. pp.1-137.

Chong FK, Lawrance KK, Lim PP, Poon MCY, Foo DCY, Lam HL, Tan RR. 2014. Planning of carbon capture storage deployment using process graph approach. Energy 76, 641-651.

Farid AM, Thompson D, Hegde P, Schoonenberg W. 2021. A Tensor-Based Formulation of Hetero-functional Graph Theory. arXiv:2101.07220v1 [cs.AI]

Mukherjee S 2014 Atlas of Shear Zone Structures in Meso-scale. Springer Geology. Cham. pp. 1124. ISBN 978-3-319-0088-6.

Mukherjee S 2019 Using graph theory to represent brittle plane networks. In: Problems and Solutions in Structural Geology and Tectonics. Billi A, Fagereng A (Eds). Developments in Structural Geology and Tectonics Book Series. Vol. 5. Series Editor: Mukherjee S. Elsevier. pp.259-272.ISSN:2542-9000. 
Mukherjee S, Bose N, Ghosh R, Dutta D, Misra AA, Kumar M, Dasgupta S, Biswas T, Joshi A and Limaye M 2020. Structural Geological Atlas. Springer. ISBN: 978-981-13-9825-4.

Misra AA and Mukherjee S. 2018 Seismic Structural Analysis. In: Misra AA, Mukherjee S. (Eds) Atlas of Structural Geological Interpretation from Seismic Images. Wiley Blackwell. ISBN: 9781-119-15832-5. pp. 15-26.

Pashin JC, Jin G, Zheng C, Chen S, McIntyre MR. 2008. Discrete Fracture Network Models for Risk Assessment of Carbon Sequestration in Coal. Final technical report. URL: file:///F:/Manjusha_25Jan2022/READY\%20TO\%20SUBMIT/ee/Pashin.pdf (Accessed on 25-Jan2022)

Ramakrishnan PV and Lakshmi T 2008 A note on the fusion of two vertices in a fuzzy graph; Tsukuba J. Math. 32, 155-164.

Sandersons DJ, Peacock DCP, Nixon CW and Rotevatn A 2018 Graph theory and the analysis of fracture networks; J. Struct. Geol. 125, 155-165. 
Tan RR, Ng DKS, Foo DCY, Aviso KB. 2010. Crisp and fuzzy integer programming models for optimal carbon sequestration retrofit in the power sector. Chemical Engineering Research and Design 88, 1580-1588.

Zadeh LA 1965 Fuzzy sets. Inf. Control 8, 338-353.

Zhang Y, Oldenburg CM, Finsterle S, Jordan P, Zhang K. 2009. Probability Estimation of CO2 Leakage Through Faults at Geologic Carbon Sequestration Sites. Energy Procedia 1, 41-46. 